\section{Living wills improve patient-physician communication}

The American Osteopathic Association, through its House of Delegates at its annual meeting in July, reaffirmed and amended the "Death: right to die" position paper. This statement says, "The decision to cease or omit treatment to permit a terminally ill patient whose death is imminent to die shall be based upon the wishes of the patient or his family or legal representative if the patient is incompetent to act on his own behalf."

As physicians, we must balance our responsibility to care for the terminally ill or brain-dead patient with the patient's or representative's wishes. However, we often find it difficult to initiate a discussion in this area before the patient becomes terminally ill. Yet, recent studies ${ }^{1}$ indicate that most patients do wish to discuss the issue with their physicians.

Living wills, also known as advance care documents, have been endorsed by health care organizations and public groups. Thirty eight states and the District of Columbia have passed living will acts enabling competent adults to determine the extent of health care they wish to receive should they become terminally ill or incompetent. Unfortunately, most living wills contain ambiguous lan- guage and lack uniform descriptions of impairment categories. Likewise, no standard definitions exist regarding the intensity of treatment.

With that in mind, Emanuel and Emanuel ${ }^{2}$ recently proposed a standard format. Called the medical directive, it specifies the patient's treatment or nontreatment preferences. Specifically, the advance care document contains four paradigmatic illness scenarios. Each one features the patient's preferred medical care interventions. The document also has a section in which a proxy decision maker may be designated. Options for organ donation are included as well.

Ideally, such a document, written in straightforward, easy-to-understand language and kept on file in the physician's office, would serve as a catalyst for improved communication between patient and physician. The physician would have a better understanding of the patient's wishes. More important, the patient will remain the captain of his or her ship.

\section{THOMAS WESLEY ALLEN, DO}

\footnotetext{
1. Kinsella TD, Stocking CB: Failed communication about life-support therapy: Silent physicians and mute patients. Am J Med 1989;86:643644 .

2. Emanuel LL, Emanuel EJ: The medical directive: A new comprehensive advance care document. JAMA 1989;261:3288-3293.
} 
NSAID-INDUCED

\section{GASTRIC MUCOSALINJURY:}

\section{THEUNDERIYING PROBIEM OFNSAID THERAPY}




\section{CHRONICNSAID USERS EXPERIENCE A DIFFERENT KIND OF ULCER}

\section{ISAIDs inhibit prostaglandins that protect the} tomach

ISAIDs relieve pain and disability for millions of Amerians by inhibiting the formation of prostaglandins. But rostaglandin depletion may jeopardize the gastric nucosa, leaving patients vulnerable to NSAID-induced yastric mucosal injury.

\section{ISAID ulcers differ from acid ulcers ${ }^{1.3}$}

he mechanism responsible for NSAID-induced gastric ulcers - prostaglandin depletion, not solely hypersecreon of acid - may explain the lack of demonstrated efficacy of conventional antiulcer agents to prevent he problem.

\begin{tabular}{|l|l|l|}
\hline ulcer site & $\begin{array}{l}\text { NSAID-induced } \\
\text { ulcer }\end{array}$ & $\begin{array}{l}\text { Acid (classic) } \\
\text { ulcer disease }\end{array}$ \\
\hline $\begin{array}{l}\text { etiology/ } \\
\text { pathogenesis }\end{array}$ & $\begin{array}{l}\text { NSAID- } \\
\text { gastric } \\
\text { associated/ } \\
\text { prostaglandin } \\
\text { (PGE) } \\
\text { depletion }\end{array}$ & $\begin{array}{l}\text { usually } \\
\text { duodenal }\end{array}$ \\
\hline symptomatology & $\begin{array}{l}\text { unknown/ } \\
\text { acid- } \\
\text { mediated } \\
\text { frequently }\end{array}$ & $\begin{array}{l}\text { usuallypain. } \\
\text { pressure, } \\
\text { and/or } \\
\text { dyspepsia }\end{array}$ \\
\hline
\end{tabular}

Eytotec replaces the prostaglandin that SSAIDs deplete

Cytotec, a synthetic analog of $P G E_{1}$, helps restore the tomach's natural defense mechanisms while NSAID herapy continues. Cytotec replaces the prostaglandin that protects the gastric mucosa. In animal studies, Cytotec has been proven to increase mucus secretion, ${ }^{4}$ increase bicarbonate secretion, and decrease $\mathrm{HCl}$ secretion. ${ }^{6.7}$

\section{Special considerations before you prescribe Cytotec \\ Cytotec is contraindicated, because of its abortifacient property, in women who are pregnant. Patients must be advised of the abortifacient property and warned not to give the drug to others. \\ Cytotec should be used in a woman of childbearing potential only if she is using effective contraceptive measures, has received oral and written warnings con- cerning the hazards of misoprostol, has had a negative serum pregnancy test within two weeks prior to begin- ning therapy, and will begin therapy only on the second or third day of the next menstrual period. See the accompanying complete prescribing information. \\ Physicians should be aware that patients may experi- ence transient diarrhea. Abdominal discomfort may persist in the absence of gastric ulceration.}

\section{Cytotec and NSAIDs-a protective partnership} All NSAIDs can cause gastric injury. ${ }^{8}$ Patients at high risk of NSAID-induced gastric injury include those over 60 , those with a history of gastric problems, and those with concomitant debilitating disease. ${ }^{2.810}$

The next time you prescribe an NSAID, remember its protective partner, Cytotec. It takes the major risk out of NSAID therapy.

\section{For NSAID patients at risk, prescribe}

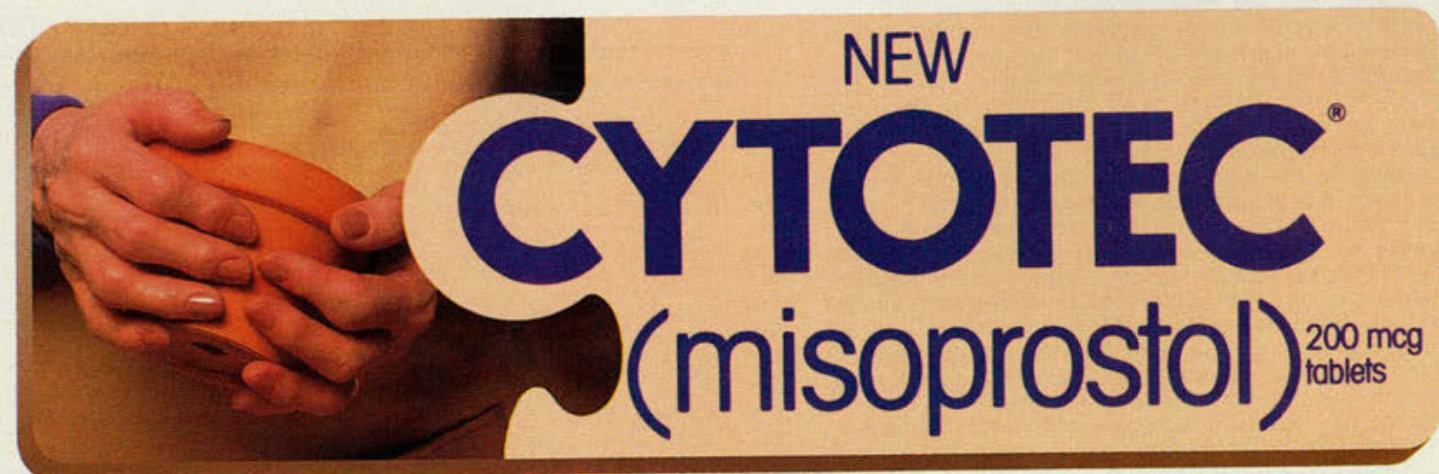

Please see adjoining page for brief summary of prescribing information. 
For NSAID patients at risk, prescribe
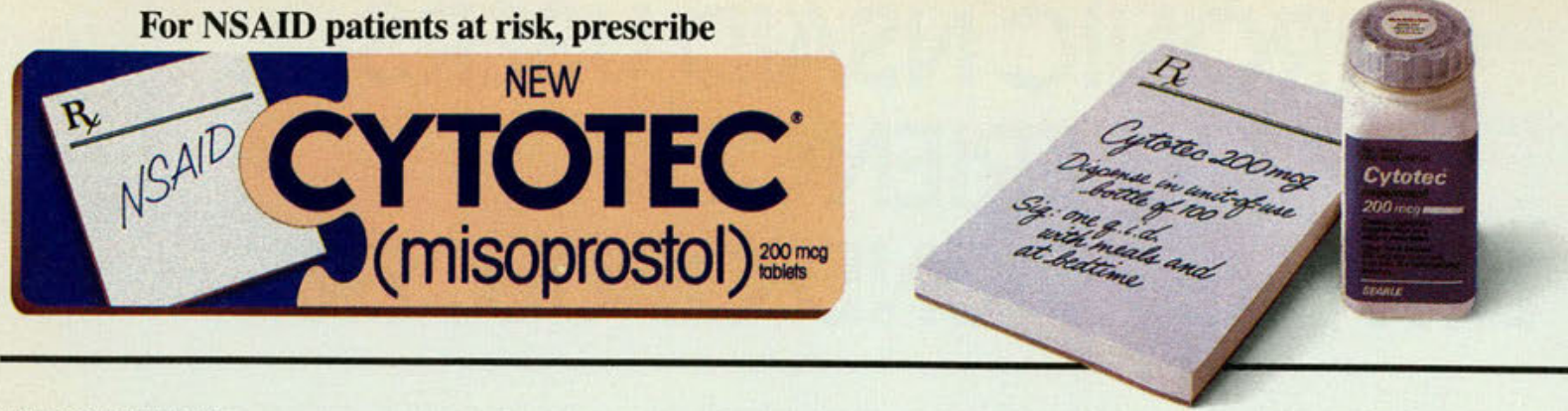

\section{BRIEF SUMMARY}

CONTRAINDICATIONS AND WARNINGS: Cytotec (misoprostol) is contraindicated, because of its abortifacient property, in women who are pregnant. (See Precautions.) Patients must be advised of the abortifacient property and warned not to give the drug to others. Cytotec should not be used in women of childbearing potential unless the patient requires nonsteroidal anti-inflammatory drug (NSAID) therapy and is at high risk of complications from gastric ulcers associated with the use of the NSAID, or is at high risk of developing gastric ulceration. In such patients, Cytotec may be prescribed if the patient

- is capable of complying with effective contraceptive measures.

- has received both oral and written warnings of the hazards of misoprostol, the risk of possible contraception failure, and the danger to other women of childbearing potential should the drug be taken by mistake.

- has had a negative serum pregnancy test within two weeks prior to beginning therapy.

- will begin Cytotec only on the second or third day of the next normal menstrual period.

INDICATIONS AND USAGE: Cytotec (misoprostol) is indicated for the prevention of NSAID (nonsteroidal anti-inflammatory drugs, including aspirin)-induced gastric ulcers in patients at high risk of complications from gastric ulcer, eg, the elderly and patients with concomitant debilitating disease, as well as patients at high risk of developing gastric ulceration, such as patients with a history of ulcer. Cytotec has not been shown to prevent duodenal ulcers in patients taking NSAIDs. Cytotec should be taken for the duration of NSAID therapy. Cytotec has been shown to prevent gastric ulcers in controlled studies of three months' duration. It had no effect, compared to placebo, on gastrointestinal pain or discomfort associated with NSAID use.

CONTRAINDICATIONS: See boxed CONTRAINDICATIONS AND WARNINGS. Cytotec should not be taken by anyone with a history of allergy to prostaglandins.

WARNINGS: See boxed CONTRAINDICATIONS AND WARNINGS.

PRECAUTIONS: Information for patients: Women of childbearing potential should be told that they must not be pregnant when Cytotec therapy is initiated, and that they must use an effective contraception method while taking Cytotec

See boxed CONTRAINDICATIONS AND WARNINGS.

Patients should be advised of the following:

Cytotec is intended for administration along with nonsteroidal antiinflammatory drugs (NSAIDs), including aspirin, to decrease the chance of developing an NSAID-induced gastric ulcer.

Cytotec should be taken only according to the directions given by a physician.

If the patient has questions about or problems with Cytotec, the physician should be contacted promptly.

THE PATIENT SHOULD NOT GIVE CYTOTEC TO ANYONE ELSE. Cytotec has been prescribed for the patient's specific condition, may not be the correct treatment for another person, and may be dangerous to the other person if she were to become pregnant

SPECIAL NOTE FOR WOMEN: Cytotec must not be used by pregnant women. Cytotec may cause miscarriage. Miscarriages caused by Cytotec may be incomplete which could lead to potentially dangerous bleeding.

Drug interactions: Cytotec has not been shown to interfere with the beneficial effects of aspirin on signs and symptoms of rheumatoid arthritis. Cytotec does not exert clinically significant effects on the absorption, blood levels, and anti-platelet effects of therapeutic doses of aspirin. Cytotec has no clinically significant effect on the kinetics of diclofenac or ibuprofen. Pharmacokinetic studies showed no drug interaction with antipyrine and propranolol when given with misoprostol.

Animal toxicity: A reversible increase in the number of normal surface gastric epithelial cells occurred in the dog, rat, and mouse. No such increase has been observed in humans administered Cytotec for up to one year.

An apparent response of the female mouse to Cytotec in long-term studies at 100 to 1000 times the human dose was hyperostosis, mainly of the medulla of sternebrae. Hyperostosis did not occur in long-term studies in the dog and rat and has not been seen in humans treated with Cytotec. Carcinogenesis, mutagenesis, impairment of fertility: Cytotec was not mutagenic in several in vitro assays, nor was it carcinogenic in rats or mice.

Misoprostol, when administered to breeding male and female rats at doses 6.25 times to 625 times the maximum recommended human therapeutic dose, produced dose-related pre- and post-implantation losses and a significant decrease in the number of live pups born at the highest dose. These findings suggest the possibility of a general adverse effect on fertility in males and females.

Pregnancy: Pregnancy Category $\mathrm{X}$ : See boxed CONTRAINDICATIONS AND WARNINGS.
Nonteratogenic effects: Cytotec may endanger pregnancy (may cause miscarriage) and thereby harm to the fetus when administered to a pregnant woman. Cytotec produces uterine contractions, uterine bleeding, and expulsion of the products of conception. Miscarriages caused by Cytotec may be incomplete. In studies in women undergoing elective termination of pregnancy during the first trimester, Cytotec caused partial or complete expulsion of the products of conception in $11 \%$ of the subjects and increased uterine bleeding in $41 \%$. If a woman is or becomes pregnant while taking this drug, the drug should be discontinued and the patient apprised of the potential hazard to the fetus.

Teratogenic effects: Cytotec is not fetotoxic or teratogenic in rats and rabbits at doses 625 and 63 times the human dose, respectively.

Nursing mothers: See Contraindications. Cytotec should not be administered to nursing mothers because the potential excretion of misoprostol acid could cause significant diarrhea in nursing infants.

Pediatric use: Safety and effectiveness in children below the age of 18 years have not been established.

ADVERSE REACTIONS:

Gastrointestinal: The most frequent gastrointestinal adverse events were diarrhea and abdominal pain. The incidence of diarrhea ranged up to $40 \%$ but averaged $13 \%$ in clinical trials. Diarrhea was dose related and usually developed early in the course of therapy (after 13 days), usually was selflimiting (often resolving after 8 days), but sometimes required discontinuation of Cytotec ( $2 \%$ of the patients). The incidence of diarrhea can be minimized by administering after meals and at bedtime, and by avoiding coadministration of Cytotec with magnesium-containing antacids.

Gynecological: Women who received Cytotec during clinical trials reported the following gynecological disorders: spotting $(0.7 \%)$, cramps $(0.6 \%)$. hypermenorrhea $(0.5 \%)$, menstrual disorder $(0.3 \%)$ and dysmenorrhea $(0.1 \%)$. Postmenopausal vaginal bleeding may be related to Cytotec administration. If it occurs, diagnostic workup should be undertaken to rule out gynecological pathology.

Elderly: There were no significant differences in the safety profile of Cytotec in approximately 500 ulcer patients who were 65 years of age or older compared with younger patients.

Additional adverse events which were reported are categorized as follows: Incidence greater than $1 \%$ : In clinical trials, the following adverse reactions were reported by more than $1 \%$ of the subjects receiving Cytotec and may be causally related to the drug: nausea $(3.2 \%)$, flatulence $(2.9 \%)$, headache $(2.4 \%)$, dyspepsia $(2.0 \%)$, vomiting $(1.3 \%)$, and constipation (1.1\%). However, there were no significant differences between the incidences of these events for Cytotec and placebo.

Causal relationship unknown: The following adverse events were infrequently reported. Causal relationships between Cytotec and these events have not been established but cannot be excluded: aches/pains, asthenia, fatigue, fever, rigors, weight changes, rash, dermatitis, alopecia, pallor, breast pain, abnormal taste, abnormal vision, conjunctivitis, deafness, tinnitus, earache, upper respiratory tract infection, bronchitis, bronchospasm, dyspnea, pneumonia, epistaxis, chest pain, edema, diaphoresis, hypotension, hypertension, arrhythmia, phlebitis, increased cardiac enzymes, syncope, GI bleeding, GI inflammation/infection, rectal disorder, abnormal hepatobiliary function, gingivitis, reflux, dysphagia, amylase increase, glycosuria, gout, increased nitrogen, increased alkaline phosphatase, polyuria, dysuria, hematuria, urinary tract infection, anxiety, change in appetite, depression, drowsiness, dizziness, thirst, impotence, loss of libido, sweating increase, neuropathy, neurosis, arthralgia, myalgia, muscle cramps, stiffness, back pain, anemia, abnormal differential, thrombocytopenia, purpura, ESR increased. Important note: Complete prescribing information should be consulted prior to use.

1/26/89 • P89-R7418V

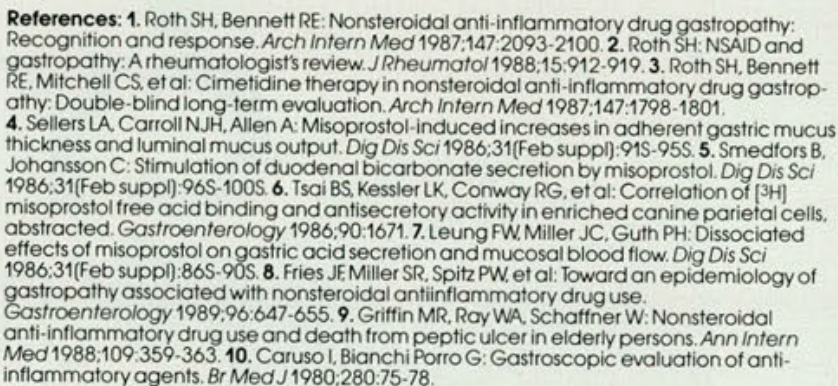

Address medical inquires to:

G.D. Searle \& Co.

Medical \& Scientific Information Department

4901 Searle Parkway

Skokie IL 60077 